\title{
CALCIFICAÇÃO NOS NÚCLEOS DA BASE NA TOMOGRAFIA COMPUTADORIZADA
}

Ao Editor - Após a leitura do artigo de Tedrus et al. ${ }^{1}$, gostaríamos de tecer alguns comentários. Os autores relatam, em amostra de 25 pacientes com calcificação nos núcleos da base, queixas ou lesões neurológicas em 23, e sinais e sintomas não-neurológicos em apenas dois casos. Na literatura, a repercussão clínica relacionada a essas calcificações tem igualmente destacado quadros n e u rológicos, principalmente desordens do movimento e distúrbios cognitivos, ou quadros psiquiátricos. No entanto, do ponto de vista neuroendócrino, é necessária atenção ao eixo hipotálamo-hipófise-gônada. Em 1997 foi publicado o caso de paciente feminina, com calcificações simétricas em núcleos da base, idiopáticas, na qual foi diagnosticado hipogonadismo hipogonadotrófico isolado ${ }^{2}$. As calcificações permaneceram estáveis em acompanhamento de 15 anos, sem evidências de doença n e u rológica. Logo após, foi detectada a mesma associação - calcificação idiopática nos núcleos da base e hipogonadismo hipogonadotrófico - em dois novos pacientes, um do sexo masculino, que apresentava disfasia, e outro no sexo feminino, sem sintomas neurológicos ${ }^{3}$. A presença das calcificações é infreqüente na observação de Tedrus et al. ${ }^{1}$ e, apesar da ocorrência não estabelecida na literatura, mais infreqüente ainda a deficiência isolada de gonadotrofinas. A baixa prevalência das duas entidades pode contribuir para o desconhecimento da origem - fortuita ou dependente - da associação. No entanto, a possibilidade de diagnóstico e intervenção terapêutica específica para o hipogonadismo valida chamar a atenção para a sua possível ocorrência frente a indivíduos com calcificação em núcleos da base em idade reprodutiva.

\section{REFERÊNCIAS}

1. Tedrus GMAS, Fonseca LC, Nogueira JrE. Calcificação nos núcleos da base na tomografia computadorizada. Arq Neuropsiquiatr 2006;64(1):104-107.

2. Pereira-Filho A, Marroni CP, Oliveira MC. Síndrome de Fahr e comprometimento do eixo gonadal. Arq Bras Endocrinol Metab 2002;46:pS46.

3. Oliveira MC, Cremonese RV. Hipogonadismo hipogonadotrófico concomitante à síndrome de Fahr. Arq Bras Endocrinol Metab 1997;41:188-90.

Miriam da Costa Oliveira Centro de Neuroendocrinologia da Santa Casa de Porto Alegre Fundação Faculdade Federal de Ciências Médicas mco@portoweb.com.br
Resposta do Autor - No artigo "Calcificação nos núcleos da base: correlação clínica em 25 pacientes con secutivos" foi discutida a possível relação entre a calcificação dos núcleos da base e as manifestações clínicas observadas nesses pacientes, e, na maioria dos casos, não foi possível estabelecer correlação entre o quadro clínico e o local envolvido na calcificação', em acordo com o referido na literatura ${ }^{2-8}$.

É possível que a associação entre hipogonadismo hipogonadotrófico e calcificação dos núcleos da base referida por Oliveira e Cremonese $(1997)^{9}$ em um caso e por Pereira-Filho et al. (2002) em dois casos, seja um achado incidental ${ }^{10}$.

Entretanto, conforme sugerido por Oliveira, na seção "correspondência" da revista Arquivos de Neuropsiquia tria, a investigação de hipogonadismo pode ser aventada em indivíduos que apresentem calcificação dos núcleos da base em idade reprodutiva.

\section{REFERÊNCIAS}

1. Tedrus GMAS; Fonseca LC; Nogueira-Junior E. Calcificação nos núcleos da base: correlação clínica em 25 pacientes consecutivos. Arq Neuropsiquiatr 2006;64: 104-107.

2. Goldscheider HG, Lischewski R, Claus D, Streibl W, Waiblinger G. Clinical, endocrinological, and computerized tomography scans for symmetrical calcification of the basal ganglia. Arc Psychiatr Nervenkr 1980;228:53-65.

3. Kazis AD. Contribution of CT scan to the diagnosis of Fahr's syndrome. Acta Neurol Scand 1985;71:206-211.

4. Manyam BV, Bhatt Moore WD, Develeschoward AB, Anderson DR, Calne DB. Bilateral striopallidodentate calcinosis: cerebrospinal fluid, imaging, and electrophysiological studies. Ann Neurol 1992;31:379-384.

5. Legido A, Zmmerman RA, Packer RJ, Bilanluk LT, Siegel K, Dángil G. Significance of basal ganglia calcification on computed tomography in children. Paediatr Neurosci 1988;14:64-70.

6. Taxer F, Haller R, Konig P. Clinical early symptoms and CT findings in Fahr syndrome. Nervenarzt 1986;57:583-588.

7. Morgante L, Trimarchi F, Benvenga S. Fahr's disease: clinical picture. Lancet 2002; 359:759.

8. Delgado-Rodrigues RN. Neurocisticercose associada a hipoparatireoidismo e doença de Fahr. Registro de um caso. Arq Neuropsiquiatr 1984;42:388-391.

9. Oliveira MC, Cremonese RV. hipogonadismo hipogonadotrófico concomitante à síndrome de Fahr. Arq Bras Endocrinol Metab 1997;41:188-190.

10. Pereira-Filho A, Marroni CP, Oliveira MC. Síndrome de Fahr e comprometimento do eixo gonadal. Arq Bras Endocrinol Metab 2002;46:pS46. 